\title{
EXTREMAL MARGINAL TRACIAL STATES IN COUPLED SYSTEMS
}

\author{
GEOFFrEy L. PrICE* AND SHÔICHIRÔ SAKAI
}

Abstract. Let $\Gamma$ be the convex set consisting of all states $\phi$ on the tensor product $B \otimes B$ of the algebra $B=M_{n}(\mathbb{C})$ of all $n \times n$ matrices over the complex numbers $\mathbb{C}$ with the property that the restrictions $\phi_{\backslash B \otimes I}$ and $\phi_{\backslash I \otimes B}$ are the unique tracial states on $B \otimes I$ and $I \otimes B$. We find necessary and sufficient conditions for such a state, called a marginal tracial state, to be extremal in $\Gamma$. We also give a characterization of those extreme points in $\Gamma$ which are pure states. We conjecture that all extremal marginal tracial states are pure states.

Mathematics subject classification (2000): 46L30, 46L06.

Key words and phrases: Pure state, trace, marginal tracial state, Schmidt decomposition.

\section{REFERENCES}

[EK] A. EKERT AND P. L. KNIGHT, Entangled quantum systems and the Schmidt decomposition, Am. J. Phys. 63 (1995), 415-423.

[P1] K. R. Parthasarathy, Extremal quantum states in coupled systems, Ann. Inst. H. Poincaré 41 (2005), 257-268.

[P2] K. R. PARThasarathy, On extremal correlations, J. Stat. Planning and Inf. 103 (2002), 73-80.

[Sa] S. SAKAI, $C^{*}$-algebras and $W^{*}$-algebras, Springer-Verlag, 1971.

[Sc] E. SCHMIDT, Zur theorie der linearen und nichtlinearen integralgleichungen, Math. Annalen 63 (1906), 433-476.

[Sz] B.Sz.-NAGY, Spektraldarstellung linearer transformationen des Hilbertschen raumes, Springer-Verlag, 1942.

[T] J. TOMiYama, Applications of Fubini type theorem to the tensor products of $C^{*}$-algebras, Tôhoku Math. J. (2) 19 (1967), 340-344.

[VW] K. G. H. Vollbrecht AND R. F. Werner, Why two qubits are special, J. Math. Phys. 41 (2000), 6772-6782.

(c) AlE I Z Zagreb

Paper No. 01-10 